\title{
Controle químico da microflora oral em pacientes fissurados labiopalatais durante o tratamento orto- dôntico-cirúrgico: estudo piloto
}

\author{
Alcion Alves SILVA*, João Luiz CARLINI**, Solena Ziemer KUSMA***
}

\begin{abstract}
Resumo
O risco de infecção é uma preocupação durante o tratamento ortodôntico-cirúrgico, principalmente em pacientes como os fissurados lábio palatais, pois as condições anatômicas e oclusais contribuem para a proliferação microbiana. Métodos manuais para o controle da microflora oral são muitas vezes insuficientes para a obtenção de uma higiene satisfatória. Técnicas alternativas, como agentes químicos, têm sido propostas como procedimentos auxiliares. O objetivo deste estudo foi comparar a eficiência de dois agentes químicos como auxiliares no controle da microflora, em pacientes ortodôntico-cirúrgicos (fissurados lábio-palatais). O delineamento da pesquisa foi um estudo exploratório, microbiológico (piloto), duplo-cego, para testar os seguintes agentes químicos: triclosan 0,03\% (Plax-Colgate Palmolive) e clorexidina digluconada 0,12\% (Duplak-Herpro/Dentsply). A amostra foi composta por 30 voluntários divididos em 3 grupos: Grupo I (controle), Grupo II (teste - clorexidina digluconada 0,12\%) e Grupo III (teste - triclosan 0,03\%). Todos os grupos receberam orientação de higiene bucal prévia à primeira coleta de material. Depois de sete dias de uso dos agentes químicos uma segunda coleta foi realizada e o uso do agente foi suspenso. Uma terceira coleta foi realizada após sete dias a partir da suspensão do agente. A técnica semiquantitativa proposta por Pilonetto e Pilonetto ${ }^{20}$ foi adotada para avaliar o crescimento de colônias bacterianas. Os resultados mostraram diferenças entre a eficácia dos agentes químicos, sendo que o Grupo II mostrou uma redução mais significativa da microflora quando comparado ao grupo III.
\end{abstract}

Palavras-chave: Controle químico. Microflora oral. Orto-cirurgia.

\section{INTRODUÇÃO E REVISÃO DA LITERATURA}

O controle da microflora oral é uma preocupação presente na Odontologia preventiva contemporânea ${ }^{25}$, principalmente relacionado às doenças orais com maior incidência, como a cárie e a doença periodontal ${ }^{18,33}$, porém extensivo às demais especialidades. O controle efetivo da placa bacte- riana é a base para a prevenção, realizada por métodos mecânicos e químicos ${ }^{19,22}$.

Entre os microorganismos freqüentes na cavidade bucal, o Streptococcus é um gênero de bactéria associado a infecções, sendo que a espécie mutans, especificamente com a doença cárie ${ }^{12}$. Sua freqüência foi observada em $85 \%$ da população

* Doutor pela Universidade Federal do Rio de Janeiro. Membro do Centro Integrado Orto-Cirúrgico.

** Doutor pela Universidade Federal do Rio de Janeiro. Professor da Universidade Federal do Paraná.

*** Mestranda em Saúde Coletiva - Londres. 
adulta $^{4}$, variando de acordo com diversos fatores, entre os quais: nível de informação sobre higiene oral, dieta e sistema imunológico ${ }^{27}$.

Existem situações complexas para se obter um satisfatório controle da microbiota, somente com procedimentos mecânicos como a escovação dos dentes. Pacientes como os fissurados labiopalatais ${ }^{13}$, ou aqueles submetidos a procedimentos ortodônticos e cirúrgicos ${ }^{2,16,17,26}$ representam exemplos. O risco de infecção durante tratamentos ortodônticocirúrgicos, devido ao período de cicatrização ocorrer com o paciente sob bloqueio intermaxilar, associado à presença de aparelhagem ortodôntica, é aumentado, devido às condições favoráveis para a ocorrência do acúmulo de placa bacteriana.

Muitos agentes químicos foram propostos como auxiliares para o controle dos microorganismos bucais $^{9,14,15,18,24}$ : agentes fenólicos (propostos por Joseph Lister em 1865 para assepsia da cavidade bucal); triclosan (bisfenol iônico de baixa toxicidade e largo espectro germicida) ${ }^{5,6,7}$; gluconato de clorexidina (germicida catiônico de largo espectro, cujo uso prolongado produz manchas marrons nas superfícies dos dentes e restaurações, além de alterações do paladar) ${ }^{1}$; soluções fluoretadas (fluoreto estanhoso a $0,05 \%$ ); agentes oxigenados (peróxido de hidrogênio a $1,5 \%$, utilizado em pacientes ortodônticos para controle de placa dental, contudo com efeitos colaterais, como irritação da mucosa) ${ }^{3}$.

Três mecanismos foram identificados como responsáveis pelo controle microbiano, através da utilização de agentes químicos ${ }^{10,23}$ :

a) Eliminação da colonização da placa na superfície do dente;

b) Redução da placa para condições de saúde, e inibição do desenvolvimento desta;

c) Mudanças na composição da placa e inibição de patologias;

\section{OBJETIVO}

O objetivo deste estudo foi testar, preliminarmente (estudo piloto), dois agentes químicos, como componentes auxiliares na rotina de higiene bucal, para o potencial controle do gênero Streptococcus, em pacientes portadores de fissuras labiopalatais, submetidos a tratamento ortodônticocirúrgico, provenientes do Centro de Assistência Integral ao Paciente Fissurado (CAIF), localizado na Cidade de Curitiba/PR, Brasil.

\section{MATERIAL E MÉTODOS Delineamento}

A presente pesquisa foi delineada como um estudo exploratório, com o objetivo de auxiliar o futuro delineamento experimental, desta forma, com amostra reduzida; duplo-cego, já que nem os microbiologistas nem os pesquisadores clínicos sabiam sobre a identificação da amostra; proposta para testar a eficácia no controle de microorganismos de dois agentes químicos:

1) Plax (Colgate Palmolive Ltda): princípio ativo, triclosan $(0,03 \%)$, fluoreto de sódio (225 ppm), Gantrez (0,20\%);

2) Duplak (Herpro/Dentsply): princípio ativo, digluconato de Clorexidina 0,12\% em solução aquosa.

\section{Amostra}

A amostra foi composta por 31 voluntários, acometidos por fissuras labiopalatais unilaterais completas, em tratamento com aparelho ortodôntico fixo, com bandas nos primeiros molares, idade entre 6 e 12 anos. Todos foram selecionados aleatoriamente no serviço do Centro de Atendimento Integral ao Fissurado Labiopalatal da cidade de Curitiba - Paraná, e concordaram com o termo de consentimento livre e esclarecido. A amostra foi dividida em três grupos:

- Grupo I (controle): recebeu apenas instrução sobre higiene oral com técnica de escovação de Stillman modificada, realizada com freqüência de 3 vezes ao dia, sem o uso de agentes químicos.

- Grupo II (teste): recebeu idêntica instrução de higiene oral do grupo I, e realizou bochecho três vezes ao dia, por 30 segundos, com $15 \mathrm{ml}$ de Duplak. 
- Grupo III (teste): recebeu idêntica instrução de higiene oral do grupo I, realizou bochecho três vezes ao dia, por 30 segundos, com $15 \mathrm{ml}$ de Plax.

Os grupos teste utilizaram os produtos por 7 dias.

\section{Metodologia}

Previamente ao uso dos agentes químicos, foi procedida, para todos os grupos, coleta salivar da superfície palatina dos incisivos superiores, através de um swab esterilizado. Justificou-se a região de coleta pela proximidade com a área de realização de enxertia óssea nestes pacientes (fissurados). Embora a fase cirúrgica não tenha sido procedida no período decorrente deste estudo, a avaliação do controle da microbiota em regiões de incisão cirúrgica possibilita avaliar o risco de infecção.

Após a coleta do material na clínica, o swab foi colocado em um tubo estéril, contendo meio de transporte Stuart, e conduzido imediatamente ao laboratório microbiológico. No laboratório, o material foi semeado em placas de agar sangue e incubado por 24 horas a $37^{\circ}$ Celsius. Após o crescimento, quatro colônias foram reisoladas em agar sangue e incubadas por mais 24 horas. Finalmente, para identificação do Streptococcus, 6 testes bioquímicos foram realizados: arginina, esculina hidrolisada, utilização dos açúcares maltose e lactose, prova da uréia e prova V.P.

Tanto as coletas como o transporte do material foram procedidos no mesmo ambientes hospitalar, e imediatamente foi iniciado o teste microbiológico, para evitar deterioração das amostras ${ }^{11}$. O tubo contendo meio de cultura foi fechado com um tampão impermeável e adequadamente etiquetado (número de identificação, data, hora, destinatário, indicação de material biológico e natureza da pesquisa). Todas as amostras tiveram sua identificação codificada, impossibilitando a tendenciosidade por parte do microbiologista ou examinadores, sendo um estudo duplo-cego.

O mesmo procedimento de coleta descrito para o controle inicial foi repetido 7 dias após o início do uso dos agentes químicos e 7 dias após a suspensão do uso dos mesmos ( 14 dias).

\section{Critérios para tabulação dos dados}

Para a quantificação do crescimento das colônias nas placas foi utilizada a técnica semiquantitativa descrita por Pilonetto e Pilonetto ${ }^{20}$, a qual adota os critérios subscritos, para três quadrantes semeados, utilizando uma alça calibrada a $0,01 \mathrm{ml}$ (1:100):

- Crescimento somente no primeiro quadrante - desenvolvimento escasso (+);

- Crescimento no segundo quadrante de 5 colônias ou mais - desenvolvimento moderado $(++)$;

- Crescimento no terceiro quadrante de 5 colônias ou mais - desenvolvimento abundante $(+++)$.

\section{Controle das variáveis intervenientes}

Todos os grupos receberam a mesma instrução prévia sobre higiene bucal, com freqüência de escovação de 3 vezes ao dia, recomendações dietéticas, e evidenciação de placa uma semana antes da primeira coleta de material.

Pacientes com 20 dentes ou menos foram descartados.

Todos os grupos receberam escovas dentais da marca Oral B, modelo Indicator, e dentifrícios da marca Colgate-Palmolive (Colgate total).

\section{DISCUSSÃO}

O delineamento do presente estudo foi elaborado para testar a eficácia de dois agentes químicos, como auxiliares no controle microbiológico da flora bucal de pacientes fissurados, submetidos a tratamento ortodôntico-cirúrgico. Além da seqüência de fases propostas para a experimentação, incluindo a composição aleatória da amostra, modelo exploratório duplo cego, coleta em região específica da cavidade bucal e transporte de amostra, a contagem do nível de bactérias foi justificada por ser este um fator predisponente para a infecção bucal ${ }^{21}$. 


\section{RESULTADOS}

\begin{tabular}{|c|c|c|c|c|}
\hline \multicolumn{9}{|c|}{ Tabela 1 - Freqüência de microorganismos - GRUPO I (controle) } \\
\hline Identific. Amostra & Coleta Inicial & Segunda Coleta & Terceira Coleta & Identificação das Colônias \\
\hline W.S.S. & +++ & ++ & S.A.H.V. \\
\hline D.S. & +++ & ++ & S.A.H.V. \\
\hline E.A.B. & +++ & +++ & S.A.H.V. \\
\hline A.S.M. & +++ & +++ & S.A.H.V. \\
\hline A.V.S. & +++ & ++ & S.A.H.V. \\
\hline M.A.S. & +++ & ++ & S.A.H.V. \\
\hline E.S. & ++ & ++ & S.A.H.V. \\
\hline B.C. & +++ & ++ & S.A.H.V. \\
\hline D.G. & +++ & ++ & ++ \\
\hline F.G.M. & +++ & ++ & S.A.H.V. \\
\hline
\end{tabular}

* Streptococcus alfa-hemolíticus do grupo víridans.

\begin{tabular}{|c|c|c|c|c|}
\hline & \multicolumn{3}{|c|}{ Tabela $\mathbf{2}$ - Freqüência de microorganismos - Grupo II (teste - Dupalk). } \\
\hline Identific. Amostra & Coleta Inicial & Segunda Coleta & Terceira Coleta & Identificação das Colônias \\
\hline L.P.F. & +++ & + & ++ & S.A.H.V. \\
\hline C.S.S. & +++ & ++ & S.A.H.V. \\
\hline K.A.F.S. & +++ & ++ & S.A.H.V. \\
\hline A.L.S. & +++ & +++ & S.A.H.V. \\
\hline E.Z. & +++ & ++ & S.A.H.V. \\
\hline A.S. & +++ & +++ & S.A.H.V. \\
\hline C.R.O. & +++ & ++ & S.A.H.V. \\
\hline S.C.S. & +++ & ++ & S.A.H.V. \\
\hline J.K. & +++ & + & S.A.H.V. \\
\hline R.C.G. & ++ & +++ & S.A.H.V. \\
\hline E.Z. & +++ & +++ & S.A.H.V. \\
\hline
\end{tabular}

\begin{tabular}{|c|c|c|c|c|}
\hline & \multicolumn{3}{|c|}{ Tabela 3 - Freqüência de microorganismos - Grupo III ( teste - Plax). } \\
\hline Identific. Amostra & Coleta Inicial & Segunda Coleta & Terceira Coleta & Identificação das Colônias \\
\hline L.F.R.S & +++ & +++ & S.A.H.V. \\
\hline J.S. & +++ & ++ & S.A.H.V. \\
\hline A.F.S. & +++ & +++ & S.A.H.V. \\
\hline L.B.F. & +++ & ++ & S.A.H.V. \\
\hline D.F.R. & +++ & +++ & S.A.H.V. \\
\hline E.M.O. & +++ & +++ & S.A.H.V. \\
\hline G.H.M.F. & +++ & +++ & S.A.H.V. \\
\hline A.P.C. & +++ & +++ & S.A.H.V. \\
\hline A.S.O. & +++ & ++ & S.A.H.V. \\
\hline R.A.L. & +++ & ++ & S.A.H.V. \\
\hline
\end{tabular}


Após os testes bioquímicos, foram identificadas cepas de Streptococcus alfa hemolíticus do grupo viridans, por meio da técnica semiquantitativa, em todas as amostras. É importante reconhecer que o gênero Streptococcus é o microorganismo responsável por vários tipos de infecções bucais, e a espécie mutans, também componente do grupo viridans, está associada à etiologia da doença cárie.

Na primeira avaliação microbiológica, foi observado um crescimento abundante de colônias nos três grupos, sugerindo a presença elevada de microorganismos no ambiente bucal. Considerando que a coleta foi realizada após a instrução de higiene bucal, o nível educacional e sócio-econômico da amostra estudada, proveniente de um serviço público municipal, provavelmente está associado com a explicação deste fenômeno. É lícito sustentar então a necessidade de incluir no protocolo de tratamento deste serviço e de similares um programa de educação sistemática para os cuidados de saúde bucal pois como observado, somente uma instrução sobre higiene bucal não obteve resultados desejáveis no controle da microbiota.

Em relação aos dois agentes químicos, os resultados obtidos após a segunda análise microbiológica sugeriram maior eficácia da clorexidina digluconada $0,12 \%$ (Duplak) na redução da microflora, após sete dias de uso contínuo, quando comparada com o triclosan 0,03\% (Plax).

Os dados resultantes da terceira análise microbiológica, realizada sete dias depois de cessada a utilização dos agentes químicos, mostraram nova elevação do desenvolvimento das colônias, sugerindo que o controle químico, como auxiliar, pode representar um procedimento efetivo na manutenção da saúde bucal dos pacientes considerados nesta amostra, bem como se adequar como uma medida preventiva no controle de infecções durante tratamentos ortodônticocirúrgicos.

\section{CONSIDERAÇÕES FINAIS}

Considerando a proposta do presente estudo piloto, as seguintes considerações finais são pertinentes na contribuição ao desenvolvimento de estudos mais abrangentes.

- Clorexidina digluconada 0.12\% (Duplak) apreesentou resultado mais efetivo para o controle da microflora bucal, quando comparada com o triclosan $0.03 \%$ (Plax);

- Aparentemente, os agentes químicos são efetivos no controle da microflora bucal, quando utilizados como agentes auxiliares em casos de pacientes acometidos por fissuras labiopalatais.

Enviado em: Agosto de 2001 Revisado e aceito: Abril de 2004

Oral microflora chemical control in cleft lip and palate patients during orthodontic-surgical treatment pilot study

\begin{abstract}
The infection risk is a preoccupation during orthodontic-surgical treatment, mainly at cleft lip and palate patients, for their anatomical and occlusal condictions, that contributs to oral microflora proliferation. Manual methods for microflora control are frequently unsuccessful to obtain a sctisfactory hygiene. Alternative techniques, like chemical agents, was proposed like auxiliary procedures. This study design was exploratoy, micro-
\end{abstract}


biological (pilot), double-blind, for test the present chemical agents: triclosan 0,03\% (Plax-Colgate-Palmolive) and digluconate clorexidin 0,12\% (Duplak-Herpro/Dentispy). The sample was composed for 30 volunteers separeted at 3 groups: Group I (control), Group II (test-digluconate clorexidin 0,12), Group III (test-triclosan $0,03 \%$ ). All groups recived buccal hygiene orientation preceding the first material colect. After 7 days of chemical agent use, a secon colect was proceeded and the agent use was stopped. A tird colect was procedeed after 7 days of agent suspension. A semiquantitative technique purposed for PILONETTO and PILONETTO (1998) was adopted to evaluate bacterial colonies growth. Results showed more significant reduction of buccal microflora at Group II when compared with Group III.

Key words: Chemical control. Oral microflora. Orto surgery.

\section{REFERÊNCIAS}

1. AXELSSON, P. Current role of pharmaceuticals in prevention of caries and periodontal disease. Int Dent J, Bristol, v. 5, no. 43, p. 473-482, 1993.

2. BERGLUND, L. J.; SMALL, C. L. Effective oral hygiene for orthodontic patients. Clin Orthod, Boulder, v. 24, no. 5, p.15-20, 1990.

3. BOYD, R. L. Effect on gingivitis of daly with 1,5\% H2O2. J Clin Periodontol, Copenhagen, no. 16, p. 557-562, 1989.

4. BRATTHALL, D. The global epidemiology of mutans streptococci. In: JOHNSON, N. W. (Ed.). Risk markers for oral diseases. Cambridge University Press, 1991. v. 1, p. 313-326.

5. CESAR-NETO, P. et al. Estudo clínico-laboratorial sobre o efeito antimicrobiano do triclosan associado ao polidimetilsiloxano. Periodontia, Fortaleza, v. 6, no. 2, p. 89-92, 1998.

6. CUMMINS, D. Zinc citrate/Triclosan: a new anti-plaque system for the control of plaque and the prevention of gingivitis: shortterm clinical and mode of action studies. J Clin Periodontol, Copenhagen, no. 18, p. 455-461, 1991.

7. CURY, J. A. Controle químico da placa dental. In: ABOPREV: promoção da saúde bucal. São Paulo: Artes Médicas, 1997. p. 313-140.

8. FURUICHI, Y. Some antiplaque and antinflamatory agents in the prevention and treatment of periodontal disease. 1999. (PhD Tesis)-Göteborg University, Faculty od Odontology, Göetborg, 1999.

9. GROSSMAN, E. Effectiveness of a pre-brushing mouthrinse under simple-trial and home-use conditions. J Clin Prev Dent, Philadelphia, v. 10, no. 1, p. 3-6, 1991.

10. JARDIM JÚNIOR, E. G.; PEDRINI, D.; XAVIER, E. P.; JARDIM, P. S. Eficácia do listeriner sobre a placa. RGO, Porto Alegre, v. 46, n. 2, p. 70-78, 1998.

11. KONEMAN, A.; SOMMERS JUNIOR, D. Diagnóstico microbiológico. 2. ed. São Paulo: Panamericana, 1993.

12. LOESCHE, W. J. Role of Streptococcus mutans in human dental decay. Annu Rev microbiol, Palo Alto, no. 50, p. 353-580, 1986.

13. MACKENZIE, W. T. et al. Comparsion of $0,12 \%$ chorhexidine mouthrinse and a essencial of mouthrinse on oral health in institutionalized mentally handicapped adults: one year results. J Periodontol, Birmingham, v. 63, p. 187-93, 1992.
14. MANDEL, I. Antimicrobial mouthrinses overwiew and updadt. J Am Dent Assoc, Chicago, v. 125, p. 2s-10s, 1994.

15. MENDES, M. M. S.; ZENÓBIO, E. G.; PEREIRA, O. L. Agentes químicos para controle de placa bacteriana. R Periodontia, [S. I.], v. 5, n. 2, p. 253-256, 1995.

16. MIZRAHI, E. Enamel demineralization following orthodontic treatment. Am J Orthod, St. Louis, v. 82, no. 1, p. 61-67, 1982.

17. MIZRAHI, E. Surface distribution of enamel opacities following orthodontic treatment. Am J Orthod, St. Louis, v. 84, no. 4, p. 323-331, 1983.

18. OLIVEIRA, W. J. Chemical control of dental plaque: adjunvant in the prevention of dental caries. CRO-MG, Belo Horizonte, v. 3, p. 27-32, 1996

19. OLIVEIRA, D. C.; ROSELL, F. L.; SAMPAIO, J. E. C.; RODRIGUES Jr., A. L. Listerine in plaque index reduction. RGO, Porto Alegre, v. 46, no. 2, p. 101-108, 1998.

20. PILONETTO, M.; PILONETTO, D. Manual de procedimentos laboratoriais em microbiologia. Pinhais: Microsience, 1998.

21. POWELL, L.V. Caries prediction: a rewiew of the literature. Comm Dent Oral Epidemiol, Copenhagen, v. 26, p. 361-371, 1998.

22. RIVERA, S.; CAVIERES, X. Efectos de los colutórios com clorhexidina al $0,1 \%$ y $0,2 \%$ en pacientes com tratamento ortodoncico. Periodontia, Fortaleza, v. 7, no. 2, p. 74-77, 1998.

23. RODRIGUES, L. G.; ZAWADZKI, P. T.; CALVETE, E. Plax effect at new bacterial plaque formation. Periodontics, Chicago, v. 8, no. 1, p. 39-43, 1999.

24. ROSING, C. K.; TOLEDO, B. E. C. Bacterial plaque chemical control: chlorexidin clinical utilization in periodontics. Periodontics, Chicago, v. 1, no. 2, p. 56-58, 1993.

25. SCHMIDSEDER, J. Odontologia estética. Trad. Stein J. P. Porto Alegre: Artes Médicas, 2000.

26. ULUKAPI, H.; KORAY, F.; EFES, B. Monitoring the caries risk of orthodontic patients. Quintessence Int, Carol Stream, v. 1, no. 28, p. $27-29,1997$

27. WALLENGREN, M. L.; JOHNSON, U.; ERICSON, D. HLADR4 and number of mutans streptococci in saliva among dental students and staff. Acta Odontol Scand, Stockholm, v. 55, no. 5, p. 296-298, 1997.

\footnotetext{
Endereço para correspondência

Alcion Alves Silva

Rua Bruno Filgueira,369/1502

Batel - Curitiba - Paraná

CEP: 80.240-220

e-mail: cioccwb@hotmail.com
} 